\title{
The COVID-19 pandemic: a catalyst to improve clinical trials
}

Prakriti Gaba and Deepak L. Bhatt $\mathbb{D}^{凶}$

The COVID-19 pandemic has provided the vital stepping stones to improve how clinical trials are conducted. We provide an overview of the major changes to the clinical trial process instigated by the pandemic and suggest ways to make these changes sustainable.

To address the public health crisis provoked by the coronavirus disease 2019 (COVID-19) pandemic, clinical trial research has occurred at a previously unimaginable pace, with $>1,100$ randomized clinical trials related to COVID-19 ongoing. Historically, certain attributes of clinical trials, including time-consuming recruitment and consent processes, tedious event adjudication and limited generalizability despite exorbitant costs, have limited their capacity to provide rapid answers to important clinical questions. Initiatives have been created to tackle these drawbacks. The Clinical Trials Transformation Initiative, for example, was founded in 2007 with the aim of generating novel ways to increase the quality and efficiency of clinical trials. However, despite the introduction of this and other promising resources, the application of innovative strategies has remained limited. Today, in a matter of just a few months, the COVID-19 pandemic has incited major changes to the infrastructure of clinical trials that have transformed and improved their potential to generate high-quality evidence efficiently.

\section{Diverse patient recruitment}

The recruitment of diverse cohorts of participants has been an ongoing challenge for principal investigators running clinical trials. Although many sites care for patients of various ethnicities and socioeconomic statuses, the individuals who follow through to trial completion are less diverse, hampering trial generalizability. In the past, the use of social media has been proposed as a way to reach a larger, more heterogeneous audience. Although this approach has been successful to some degree, modern-day trials continue to struggle to recruit diverse patient participants. This issue becomes particularly important for medical conditions, such as COVID-19, that affect patients unevenly according to race, sex and economic background ${ }^{1}$.

Amidst the COVID-19 pandemic, both social media and digital health platforms have been leveraged in novel ways to recruit heterogeneous cohorts of participants. For example, a randomized clinical trial of hydroxychloroquine as post-exposure prophylaxis for
COVID-19 used social media outreach to recruit $>800$ patients, $50 \%$ of whom were female, in $<2$ months. World Without COVID, a novel public health tool by Clara Health, is recruiting participants for a variety of clinical trials online. Another web-based effort through PCORnet, Healthcare Worker Exposure Response \& Outcomes (HERO), is enrolling health-care workers in clinical trials related to COVID-19. To date, HERO has impressively enrolled $>16,000$ health-care workers and connected many to appropriate ongoing clinical trials.

\section{Universal remote consent}

After identifying and recruiting patients suitable for a clinical trial, difficulties in obtaining consent can impede enrolment. This issue is only magnified when clinical equipoise regarding the efficacy and safety of a specific therapy is eroded, owing to the adoption of approved medications for off-label indications, such as during a pandemic. To address this hurdle, on 18 March 2020, the FDA adopted contemporary approaches to consent ${ }^{2}$. Specifically, the FDA recommended telephone calls or video visits, in which the participant verbally acknowledges their desire to be part of the trial and then either directly signs or has a witness sign the form and e-mail a photograph of it, as an alternative form of consent ${ }^{2}$. Ongoing trials are using these electronic methods and have been able to obtain patient consent more easily.

\section{Telemedical monitoring and trial visits}

As a result of the \#StayAtHome requirements, site closures and restrictions on travel implemented during the pandemic, onsite monitoring programmes and in-person visits have been converted to remote monitoring programmes. Instead of meeting with health-care practitioners in person, patient-centric technologies, including Zoom and Webex, are being used to improve efficiency and safety while maintaining high monitoring standards ${ }^{2}$. In cases in which in-person visits are required, home health-care visits are being implemented with appropriate personal protective equipment ${ }^{2}$. Although the results of many trials are still pending, making it too soon to form definitive conclusions on the 
efficacy of these adjusted practices, they hold promise in decreasing dropout rates and improving short-term and long-term monitoring.

The use of remote monitoring is especially important for the continuation of non-COVID-19 clinical trials. In cardiology, which has relied heavily on clinical trials for practice guidance, among 892 pre-pandemic clinical trials evaluating a range of cardiovascular therapies, nearly all were put on hold during the pandemic $\mathrm{c}^{3,4}$. Understanding the detrimental downstream sequelae of these delays, the FDA encouraged flexible ways of restarting trial monitoring efforts through remote visits $^{2,5,6}$. For some trial sponsors, setting up the infrastructure for remote monitoring was possible despite an interruption in the study period ${ }^{7}$. For others, these adjustments have not been financially feasible, unfortunately resulting in the termination of the clinical trials altogether8.

\section{Standardizing remote event adjudication}

In-person ascertainment of trial end points has traditionally been the gold standard for recording accurate and precise outcomes for clinical trials. As with consent and monitoring, ascertainment and adjudication of clinical end points is being performed remotely during the pandemic ${ }^{2}$. E-mails, telephone calls and text messages have replaced in-person visits to ascertain the occurrence of clinical events. Online searches of obituaries are being performed when all else fails to determine a patient's vital status. The combination of efforts has been substantially less expensive than in-person adjudication and raises the question of whether in-person ascertainment of events is needed at all.

Whether centrally adjudicated end points are superior to investigator-reported outcomes has long been a controversial point, even before this pandemic ${ }^{9}$. The high costs, substantial time burden and conflicting data on the efficacy of using centrally adjudicated end points have raised questions about their necessity as advances are made in electronic medical record data recording.

\section{Novel and efficient trial designs}

A major drawback of clinical trials is their cost. The COVID-19 pandemic has encouraged the implementation of more innovative trial designs. For example, the NIAID-sponsored Adaptive COVID-19 Treatment Trial (NCT04280705) and the WHO-sponsored SOLIDARITY trial (ISRCTN83971151) are leveraging adaptive clinical trial designs, in which multiple, prespecified, investigational therapies can be compared with placebo to identify subgroups of patients who respond best to them ${ }^{10}$. Pragmatic trial designs have also been proposed to evaluate therapies in a wider array of patients with the disease and will help to increase the success of clinical trials.

Amidst these changes, concerns have emerged about trial quality and have called into question whether the rapidity of research performed during the pandemic has been detrimental. Several ongoing clinical trials lack control groups, have poorly defined end points, lack generalizability to those of a lower socioeconomic status or were designed too early in the pathophysiological course of the disease to result in substantive recommendations. In the pre-COVID-19 era, many of these issues would have constituted noncompliance with the FDA's Code of Federal Regulations and compromised the validity of the study results ${ }^{8}$. Tempering expectations when the results of these studies are published will be crucial. Nonetheless, we do not believe that these concerns should impede the use of creative and innovative approaches that increase the efficiency of the clinical trials process. Instead, they should provide the much-needed impetus for further improvements.

Going forward, we must cement the lessons learned from the pandemic to create a meaningful change in the way in which clinical trials are performed. Three tangible ways to accomplish this aim are as follows:

- Regulatory agencies should consider making remote consent, telemedical monitoring and remote event ascertainment permanent for all clinical trials, whether or not they relate to COVID-19.

- Governments should improve access to web-based technologies to ensure the participation of individuals with lower socioeconomic status and that these individuals follow through in clinical trials that are propagated by social media or online databases.

- Non-profit and industry sponsors of trials should incentivize trialists to incorporate adaptive or pragmatic trial designs, when possible, to increase the efficiency, generalizability and success of clinical trials.

\section{Conclusions}

We are at a critical juncture for clinical trials. COVID-19 has been a catalyst for positive change. We must now use the important lessons on trial recruitment, monitoring and innovation learned during these unprecedented times to build a more innovative and integrated research platform for the future.

1. Wadhera, R. K. et al. Variation in COVID-19 hospitalizations and deaths across New York City boroughs. JAMA 323, 2192-2195 (2020).

2. FDA. FDA Guidance on Conduct of Clinical Trials of Medical Products during COVID-19 Public Health Emergency https:// www.fda.gov/media/136238/download (2020).

3. Nicholls, M. COVID-19 and cardiovascular disease. Eur. Heart J. 41, 2727-2729 (2020).

4. Selvaraj, S. et al. The landscape of cardiovascular clinical trials in the United States initiated before and during COVID-19. JAHA https://doi.org/10.1161/JAHA. 120.018274 (2020).

5. Roux, S. \& Bhatt, D. L. Self-treatment for acute coronary syndrome: why not? Eur. Heart J. 41, 2144-2145 (2020).

6. Bhatt, A. S. et al. Fewer hospitalizations for acute cardiovascular conditions during the COVID-19 pandemic. J. Am. Coll. Cardiol. 76, 280-288 (2020).

7. Wang, X. \& Bhatt, D. L. COVID-19: an unintended force for medical revolution? J. Invasive Cardiol. 32, 81-82 (2020)

8. Bagiella, E., Bhatt, D. L. \& Gaudino, M. The consequences of the COVID-19 pandemic on non-COVID-19 clinical trials. J. Am. Coll. Cardiol. 76, 342-345 (2020).

9. Jatene, T. et al. Investigator-reported bleeding versus post hoc adjudication of bleeding: lessons from the CHAMPION PHOENIX trial. J. Am. Coll. Cardiol. 67, 596-598 (2016).

10. Bhatt, D. L. \& Mehta, C. Adaptive designs for clinical trials. N. Engl. J. Med. 375, 65-74 (2016).

\section{Competing interests}

D.L.B. discloses the following relationships. Advisory Board: Cardax, CellProthera, Cereno Scientific, Elsevier Practice Update Cardiology, Level Ex, Medscape Cardiology, PhaseBio, PLx Pharma, Regado Biosciences. Board of Directors: Boston VA Research Institute, Society of Cardiovascular Patient Care, TobeSoft. Chair: AHA Quality Oversight Committee. Data Monitoring Committees: Baim Institute for Clinical Research (formerly Harvard Clinical Research Institute, for the PORTICO trial, funded by St. Jude Medical, now Abbott), Cleveland Clinic (including for the ExCEED trial, funded by Edwards), Contego Medical (Chair, PERFORMANCE 2), Duke 
Clinical Research Institute, Mayo Clinic, Mount Sinai School of Medicine (for the ENVISAGE trial, funded by Daiichi Sankyo), Population Health Research Institute. Honoraria: ACC (Senior Associate Editor, Clinical Trials and News, ACC.org; Vice-Chair, ACC Accreditation Committee), Baim Institute for Clinical Research (formerly Harvard Clinical Research Institute; RE-DUAL PCI clinical trial steering committee funded by Boehringer Ingelheim; AEGIS-II executive committee funded by CSL Behring), Belvoir Publications (Editor in Chief, Harvard Heart Letter), Duke Clinical Research Institute (clinical trial steering committees, including for the PRONOUNCE trial, funded by Ferring Pharmaceuticals), HMP Global (Editor in Chief, Journal of Invasive Cardiology), Journal of the American College of Cardiology (Guest Editor; Associate Editor), K2P (Co-Chair, interdisciplinary curriculum), Level Ex, Medtelligence/ReachMD (CME steering committees), MJH Life Sciences, Population Health Research Institute (for the COMPASS operations committee, publications committee, steering committee, and USA national co-leader, funded by Bayer), Slack Publications (Chief Medica Editor, Cardiology Today's Intervention), Society of Cardiovascular Patient Care (Secretary/Treasurer), WebMD (CME steering committees). Other: Clinical Cardiology (Deputy Editor), NCDR-ACTION Registry Steering Committee (Chair), VA CART Research and Publications Committee (Chair).
Research Funding: Abbott, Afimmune, Amarin, Amgen, AstraZeneca, Bayer, Boehringer Ingelheim, Bristol-Myers Squibb, Cardax, Chiesi, CSL Behring, Eisai, Ethicon, Ferring Pharmaceuticals, Forest Laboratories, Fractyl, Idorsia, Ironwood, Ischemix, Lexicon, Lilly, Medtronic, Pfizer, PhaseBio, PLx Pharma, Regeneron, Roche, Sanofi Aventis, Synaptic, The Medicines Company. Royalties: Elsevier (Editor, Cardiovascular Intervention: A Companion to Braunwald's Heart Disease). Site Co-Investigator: Biotronik, Boston Scientific, CSI, St. Jude Medical (now Abbott), Svelte. Trustee: American College of Cardiology. Unfunded Research: FlowCo, Merck, Novo Nordisk, Takeda. P.G. declares no competing interests.

\section{RELATED LINKS}

Clinical Trials Transformation Initiative: https://www.ctti-clinicaltrials.org/ Code of Federal Regulations: https://www.fda.gov/medical-devices/

medical-device-databases/code-federal-regulations-title-21-food-and-drugs Healthcare Worker Exposure Response \& Outcomes: https://

heroesresearch.org/

World Without COVID: https://www.clarahealth.com/covid19 